\title{
Exercise capacity and muscle strength and risk of vascular disease and arrhythmia in 1.1 million young Swedish men: cohort study
}

\author{
Kasper Andersen, ${ }^{1}$ Finn Rasmussen, ${ }^{2}$ Claes Held, ${ }^{1}$ Martin Neovius, ${ }^{3}$ Per Tynelius, ${ }^{2}$ Johan Sundström ${ }^{1}$
}

${ }^{1}$ Department of Medical

Sciences and Uppsala Clinical

Research Center, Uppsala

University Hospital, Uppsala,

SE-751 85, Sweden

${ }^{2}$ Child and Adolescent Public

Health Epidemiology Unit,

Department of Public Health

Sciences, Karolinska Institutet,

Stockholm, Sweden

${ }^{3}$ Clinical Epidemiology Unit,

Department of Medicine,

Karolinska Institutet, Stockholm,

Sweden

Correspondence to: $\mathrm{K}$ Andersen

kasper.andersen@medsci.uu.se

Additional material is published

online only. To view please visit

the journal online (http://dx.doi.

org/10.1136/bmj.h4543)

Cite this as: $B M J$ 2015;351:h4543

doi: $10.1136 /$ bmj.h4543

Accepted: 14 August 2015

\begin{abstract}
OBJECTIVE

To investigate the associations of exercise capacity and muscle strength in late adolescence with risk of vascular disease and arrhythmia.
\end{abstract}

DESIGN

Cohort study.

SETTING

General population in Sweden.

\section{PARTICIPANTS}

1.1 million men who participated in mandatory military conscription between 1 August 1972 and 31 December 1995, at a median age of 18.2 years. Participants were followed until 31 December 2010.

\section{MAIN OUTCOMES}

Associations between exercise capacity and muscle strength with risk of vascular disease and subgroups (ischaemic heart disease, heart failure, stroke, and cardiovascular death) and risk of arrhythmia and subgroups (atrial fibrillation or flutter, bradyarrhythmia, supraventricular tachycardia, and ventricular arrhythmia or sudden cardiac death). Maximum exercise capacity was estimated by the ergometer bicycle test, and muscle strength was measured as handgrip strength by a hand dynamometer. High exercise capacity or muscle strength was deemed as above the median level.

RESULTS

During a median follow-up of 26.3 years, 26088 vascular disease events and 17312 arrhythmia events were recorded. Exercise capacity was inversely associated with risk of vascular disease and its subgroups. Muscle strength was also inversely associated with vascular disease risk, driven by associations of higher muscle strength with lower risk of heart failure and cardiovascular death. Exercise capacity had a $U$ shaped association with risk of

\section{WHAT IS ALREADY KNOWN ON THIS TOPIC}

High exercise capacity and muscle strength have been associated with a reduced risk of cardiovascular disease and mortality, but exercise could increase the risk of arrhythmia

Most studies have investigated this association in only middle aged and older populations, who have a high risk of bias owing to reverse causation

\section{WHAT THIS STUDY ADDS}

High exercise capacity and muscle strength in late adolescence could be associated with a reduced risk of subsequent vascular disease

Exercise capacity seems to have a U shaped association with arrhythmia, driven by a direct association with risk of atrial fibrillation and a $U$ shaped association with bradyarrhythmia

arrhythmia, driven by a direct association with risk of atrial fibrillation and a $U$ shaped association with bradyarrhythmia. Higher muscle strength was associated with lower risk of arrhythmia (specifically, lower risk of bradyarrhythmia and ventricular arrhythmia). The combination of high exercise capacity and high muscle strength was associated with a hazard ratio of 0.67 ( $95 \%$ confidence interval 0.65 to 0.70 ) for vascular events and 0.92 (0.88 to 0.97 ) for arrhythmia compared with the combination of low exercise capacity and low muscle strength.

\section{CONCLUSIONS}

Exercise capacity and muscle strength in late adolescence are independently and jointly associated with long term risk of vascular disease and arrhythmia. The health benefit of lower risk of vascular events with higher exercise capacity was not outweighed by higher risk of arrhythmia.

\section{Introduction}

Although prevention and treatment of cardiovascular diseases have improved for decades, the proportion of cardiovascular causes of death is increasing owing to the increased longevity of the global population. ${ }^{1}$ The incidence of arrhythmia rises with age, and increased longevity has also raised the burden of these diseases. ${ }^{2}$

While physical activity and high exercise capacity prevents vascular disease, ${ }^{3-6}$ strenuous exercise could induce life threatening ventricular arrhythmia in athletes with pre-existing heart disease. ${ }^{7}$ Furthermore, an increased risk of atrial fibrillation and bradyarrhythmia has been observed in athletes. ${ }^{8-12}$ Several lines of evidence point towards a causal role of exercise for the development of arrhythmia, including substrate, modulator, and trigger mechanisms. ${ }^{8}$

We hypothesised that exercise capacity and muscle strength are each directly related to the risk of subsequent arrhythmia and inversely related to the risk of subsequent vascular disease. Many previous studies have suggested such associations in middle aged or older people, but these studies have a high risk of bias owing to reverse causality. To minimise such bias, we investigated these associations in a prospective cohort of 1.1 million Swedish young men examined at mandatory military conscription in 1972-95.

\section{Methods}

\section{Sample}

This cohort study used data from all Swedish men who underwent conscription between 1 August 1972 and 31 December 1995. During that period, military conscription for men was mandatory in Sweden, and only 2-3\% 
did not undergo conscription (mainly because of severe disease or handicap). The conscriptions were performed in a standardised approach, and a total of 1274238 men were enrolled in the cohort. Conscripts had a median age of 18.2 years (10th percentile, 17.8 years; 90th percentile, 18.9 years).

We excluded all observations with missing data on the key variables: maximum exercise capacity $(\mathrm{n}=30200,2 \%)$, muscle strength $(\mathrm{n}=5118,0.4 \%)$, and conscription date $(n=78799,6 \%)$. Additionally, we excluded people who emigrated before conscription ( $n=19518,2 \%$ ), and men with a history of cardiovascular disease ( $n=3694,0.2 \%$; ICD-10 (international classification of diseases, 10th revision) code I.00-99 or similar ICD-8/ICD-9 code). Because only a small number of observations had missing data after this procedure, and missing data were assumed to be mainly due to administrative reasons, we limited all analyses to observations with complete data. This excluded another 7554 (0.5\%) men, resulting in a sample of 1126899 individuals available for analysis.

\section{Baseline examinations}

The available protocol from 2001 is similar to the examination years during the study period. We used an ergometer bicycle test to estimate participants' maximum exercise capacity (measured in watts). After five minutes of bicycling at 60-70 revolutions per minute with minimal load, the load was gradually increased by $25 \mathrm{~W}$ per minute and the conscript continued to bicycle to exhaustion. If the conscript did not obtain a maximum heart rate higher than 180 beats per minute, the instructor decided whether the conscript should be retested. We found a minor shift in the distribution of maximum exercise capacity in August 1984 (probably due to a minor change of examination protocol), and the observations in groups before and after August 1984 were standardised to the whole sample mean and standard deviation.

Of the available measures of muscle strength, we used handgrip strength measured by a hand dynamometer (in newtons). Handgrip strength has shown good correlation with lean body mass, ${ }^{13-15}$ has previously been investigated in relation to risk of cardiovascular disease, ${ }^{16}$ and is the strength measure least correlated with body weight in this cohort. Height and weight were measured, and after five to 10 minutes of rest, supine systolic and diastolic blood pressure was measured.

\section{Follow-up and outcome parameters}

Using unique numbers in Swedish national registration, we linked the military service conscription register to the Swedish national patient register, Swedish causes of death register, and Statistics Sweden registers of emigration and education level. All registers cover the whole population of Sweden. Participants were followed until 31 December 2010, and were considered at risk until the first of the following dates: the outcome under study, death, emigration, or end of follow-up.

Using these registries, we defined two primary outcomes: vascular disease (all ICD-10 codes mentioned in subgroup outcomes) and arrhythmia (all ICD-10 codes mentioned in subgroup outcomes, plus I47.9). For vascular disease, the subgroup outcomes were ischaemic heart disease (I20.0-I25.9), heart failure (I11.0; I50.0-I50.9), stroke (I60.0-I60.9; I61.0-I61.9; I63.0-I63.9; I64.0-I64.9), and cardiovascular death (I00-I99). For arrhythmia, the subgroup outcomes were atrial fibrillation or flutter (I48.9), bradyarrhythmia (I44.1; I44.2; I45.2; I45.3; I45.9; I49.5), supraventricular arrhythmia (I45.6; I47.1), and ventricular arrhythmia or sudden cardiac death (I46.0; I46.1, I46.9; I47.0; I47.2; I49.0; R96.0). Corresponding codes for ICD-9 and ICD-8 were used. The web appendix lists all the ICD codes used.

\section{Statistical analyses}

We used Cox proportional hazards models to examine the associations of the exposures muscle strength and exercise capacity with risk of arrhythmia or vascular disease. Each outcome was analysed in a separate model. We assessed the proportional hazards assumptions by inspecting log-log plots for all outcomes, finding no obvious violation of the assumption.

With directed acyclic graphs (web fig 1), two models were identified to evaluate total and direct effects. Model A evaluated the total effect, and was adjusted for age, conscription date, region, education level, height, and muscle strength or exercise capacity (muscle strength adjusted for exercise capacity, and vice versa). Model B evaluated the direct effect and adjusted to the same factors as model A, plus systolic and diastolic blood pressure, weight and ischaemic heart disease for arrhythmia outcomes only.

To assess the nature of the associations, we used multivariable regression spline models (a piecewise fitting of polynomial equations) with up to four degrees of freedom allowed for the exposure variable, body weight, and height (and one degree of freedom for the other covariates). Knots were placed at the 25th, 50th and 75 th centiles. We investigated interactions between the two main exposures and interactions between them and the continuous baseline variables using the general multivariable fractional interaction approach. ${ }^{17} \mathrm{We}$ investigated interactions between the main exposures and categorical covariates using multiplicative factors and likelihood ratio tests. After inspection of all the significant interactions (web figs 2-4), these interactions were regarded as clinically irrelevant and produced by the large sample size. In addition to these interaction analyses, we described joint effects of exercise capacity and muscle strength by constructing a four-group variable with combinations of maximum exercise capacity and muscle strength dichotomised by the median.

To describe the incidence of outcomes in absolute terms, we also analysed risk of outcomes by fifths of exposures. Stata 13 (StataCorp) was used for all calculations, and two tailed 95\% confidence intervals were used.

\section{Patient involvement}

No patients were involved in setting the research question or the outcome measures, nor were they involved in recruitment, or the design and implementation of the 
study. There are no plans to involve patients in dissemination.

\section{Results}

Participants were followed until a median age of 44.6 years (median time at risk 26.3 years). This follow-up resulted in 29.8 million person years at risk. A total of 33089 people died during follow-up. Table 1 shows the baseline characteristics.

\section{Vascular disease}

During follow-up, we identified 26088 admissions to hospital for vascular disease (ischaemic heart disease, 12188; heart failure, 3949; stroke, 7350; cardiovascular death, 5873; one person could contribute to more than one subgroup endpoint). Figure 1 shows cumulative incidence of vascular disease. We observed an inverse association between exercise capacity and risk of vascular disease, with a more pronounced association after adjusting for blood pressure and weight (fig 2, web table 1, and web fig 5). The association was of similar strength with all of the subgroup endpoints: ischaemic heart disease, heart failure, stroke, and cardiovascular death (fig 3, web table 2, and web fig 5).

Similarly, we found an inverse association of muscle strength with the risk of vascular disease, although of smaller magnitude than that of exercise capacity (fig 2). Again, associations were more pronounced in models adjusting for blood pressure and weight than in those without these covariates (web tables 1 and 2, and web fig 5). The associations with cardiovascular death and heart failure were stronger than those with stroke and ischaemic heart disease (fig 3 and web table 1).

We saw no evidence of a deviation from a combined effect of exercise capacity and muscle strength (fig 1 and table 2). The combination of high exercise capacity and high muscle strength was associated with a 33\% lower risk of vascular events than the combination of low exercise capacity and low muscle strength (table 2).

\section{Arrhythmia}

During follow-up, we identified 17312 arrhythmia events (atrial fibrillation or flutter, 9668; bradyarrhythmia, 1384; supraventricular tachycardia, 3278; ventricular arrhythmia or sudden cardiac death, 1630; unspecified arrhythmia, 1352). Figure 1 shows cumulative incidence of arrhythmia, which indicates that arrhythmia on average occurred at a younger age than vascular disease events. We found a U shaped association of exercise capacity with risk of arrhythmia. The association was similar after additionally adjusting for blood pressure, weight, and ischaemic heart disease (fig 2, web table 1, and web fig 6). This pattern was driven by an association of higher exercise capacity with higher risk of atrial fibrillation or flutter and by a U shaped association with bradyarrhythmia (fig 4 and web table 3). We saw no associations between exercise capacity and supraventricular or ventricular arrhythmia or sudden cardiac death (fig 4, web table 3, and web fig 7).

Higher muscle strength was associated with lower risk of arrhythmia. This association was more pronounced after adjusting for weight, blood pressure and ischaemic heart disease (web tables 1 and 3 and web fig 6). The main associations were of higher muscle strength with lower risk of bradyarrhythmia and ventricular arrhythmia or cardiac arrest (fig 4, web table 2, and web fig 7). No associations of muscle strength with atrial fibrillation or flutter or supraventricular arrhythmia were found.

There was no evidence of a deviation from a combined effect of exercise capacity and muscle strength (table 2). The combination of high exercise capacity and high muscle strength for model A (total effect) was associated with a $5 \%$ higher risk of arrhythmia. However, additional adjustment for systolic and diastolic blood pressure, weight, and ischaemic heart disease resulted in an $8 \%$ lower risk of arrhythmia than the combination of low exercise capacity and low muscle strength (table 2).

\begin{tabular}{|c|c|c|c|c|c|}
\hline & $\begin{array}{l}\text { Total sample } \\
(n=1122255)\end{array}$ & $\begin{array}{l}\text { Low exercise capacity } \\
\text { and low muscle } \\
\text { strength ( } n=326462)\end{array}$ & $\begin{array}{l}\text { Low exercise capacity } \\
\text { and high muscle } \\
\text { strength ( } n=246767)\end{array}$ & $\begin{array}{l}\text { High exercise capacity } \\
\text { and low muscle } \\
\text { strength }(n=218156)\end{array}$ & $\begin{array}{l}\text { High exercise capacity } \\
\text { and high muscle } \\
\text { strength ( } n=330870)\end{array}$ \\
\hline Age at conscription (years) & $18.3(0.7)$ & $18.3(0.8)$ & $18.4(0.9)$ & $18.2(0.6)$ & $18.3(0.6)$ \\
\hline Height $(\mathrm{cm})$ & $179(7)$ & $176(6)$ & $179(6)$ & $178(6)$ & $181(6)$ \\
\hline Weight (kg) & $70(10)$ & $64(9)$ & $70(10)$ & $69(8)$ & $75(9)$ \\
\hline Muscle strength, handgrip (N) & $616(98)$ & $529(57)$ & $681(62)$ & $547(47)$ & $701(68)$ \\
\hline Exercise capacity (W) & $261(47)$ & $220(26)$ & $228(22)$ & $292(29)$ & $301(34)$ \\
\hline Systolic blood pressure & $129(11)$ & $127(11)$ & $128(11)$ & $129(11)$ & $130(11)$ \\
\hline Diastolic blood pressure & $67(10)$ & $67(10)$ & $68(10)$ & $66(11)$ & $66(10)$ \\
\hline \multicolumn{6}{|c|}{ Educational level (no (\%) of participants)* } \\
\hline Primary school $<9$ years & $5402(0.5)$ & $2189(0.7)$ & $1953(0.8)$ & $403(0.2)$ & $816(0.3)$ \\
\hline Primary school 9 years & $138495(12.2)$ & $48465(15.0)$ & $45764(18.8)$ & $14203(6.6)$ & $28451(8.7)$ \\
\hline Secondary school $<2$ years & $420181(37.0)$ & $126870(39.3)$ & $108662(44.5)$ & $64590(29.8)$ & $113354(34.5)$ \\
\hline Secondary school 2-3 years & $178870(15.8)$ & $50320(15.6)$ & $33654(13.8)$ & $37009(17.1)$ & $54116(16.5)$ \\
\hline Higher education $>2$ years & $172240(15.1)$ & $42151(13.1)$ & $27737(11.4)$ & $39960(18.5)$ & $58397(17.8)$ \\
\hline Higher education $>3$ years & $202055(17.8)$ & $48923(15.1)$ & $24549(10.0)$ & $55415(25.6)$ & $67920(20.7)$ \\
\hline $\mathrm{PhD}$ & $16605(1.5)$ & $4201(1.3)$ & $1637(0.7)$ & $5031(2.3)$ & $5310(1.6)$ \\
\hline
\end{tabular}




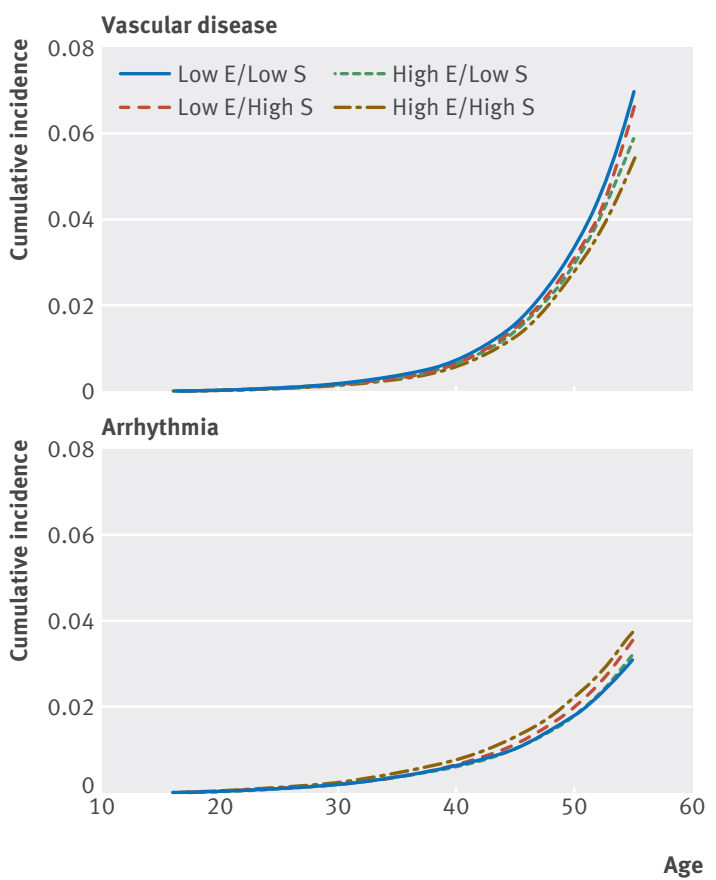

Fig 1 | Cumulative hazard estimates of vascular disease and arrhythmia, by joint groups of exercise capacity and muscle strength (defined as high or low by median values). $\mathrm{E}=$ exercise capacity; $\mathrm{S}=$ muscle strength

\section{Discussion}

In this cohort of 1.1 million men, we observed inverse independent associations of exercise capacity and muscle
Principal findings

strength in late adolescence with risk of subsequent vascular disease. There was also a U shaped association of exercise capacity with arrhythmia. The joint associations of high exercise capacity and high muscle strength with a reduced risk of vascular disease was pronounced, by comparison with low exercise capacity and low muscle strength. However, we observed weak joint associations with the risk of arrhythmia.

\section{Strengths and limitations of study}

The most obvious strength of this study was the large number of participants, which included almost all Swedish men over 25 years. Furthermore, the cohort was unique by including directly measured exercise capacity and muscle strength in a large population. By the use of unique national registration numbers and population based registers, the loss of follow-up was limited to emigrated people.

We used data from the national patient register of hospital discharges. The validity of the register is generally good, with increasing precision over the past few decades. ${ }^{18}$ Positive predictive values were $98-100 \%$ for myocardial infarction, 95\% for angina pectoris, $82-88 \%$ for heart failure, $69-98 \%$ for stroke, and $93-97 \%$ for atrial fibrillation. ${ }^{1819}$ Furthermore, we used the Swedish cause of death register to obtain information of cardiovascular death. Although the register is not regarded as precise as the inpatient register, our very large study sample should have minimised bias from randomly misclassified death certificates. We would expect an even better precision in this study sample of young men, with a reduced occurrence of comorbidities
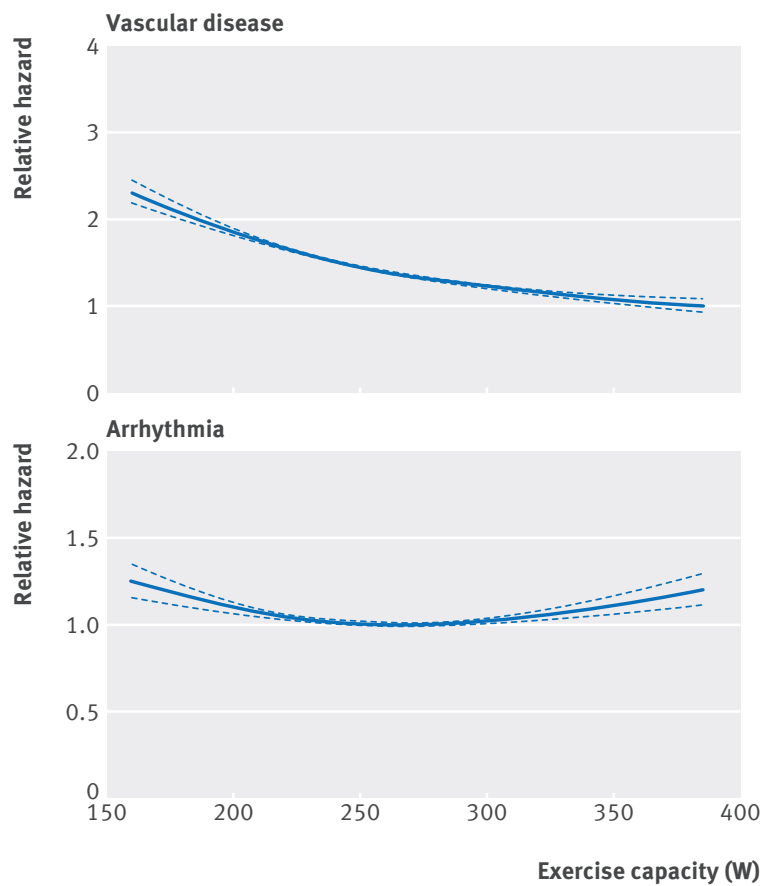

Fig 2 Relations of exercise capacity and muscle strength to risk of vascular disease and arrhythmia. Solid line=relative hazards; dashed lines $=95 \%$ confidence interval limits. Lines are based on a multivariable regression spline of Cox proportional hazards. Model B (direct effect) is shown, adjusted for age, conscription date, region, education level, height, and muscle strength or exercise capacity (muscle strength adjusted for exercise capacity, and vice versa); arrhythmia outcomes were additionally adjusted for ischaemic heart disease. Only observations between 1 and 99 percentiles are shown 

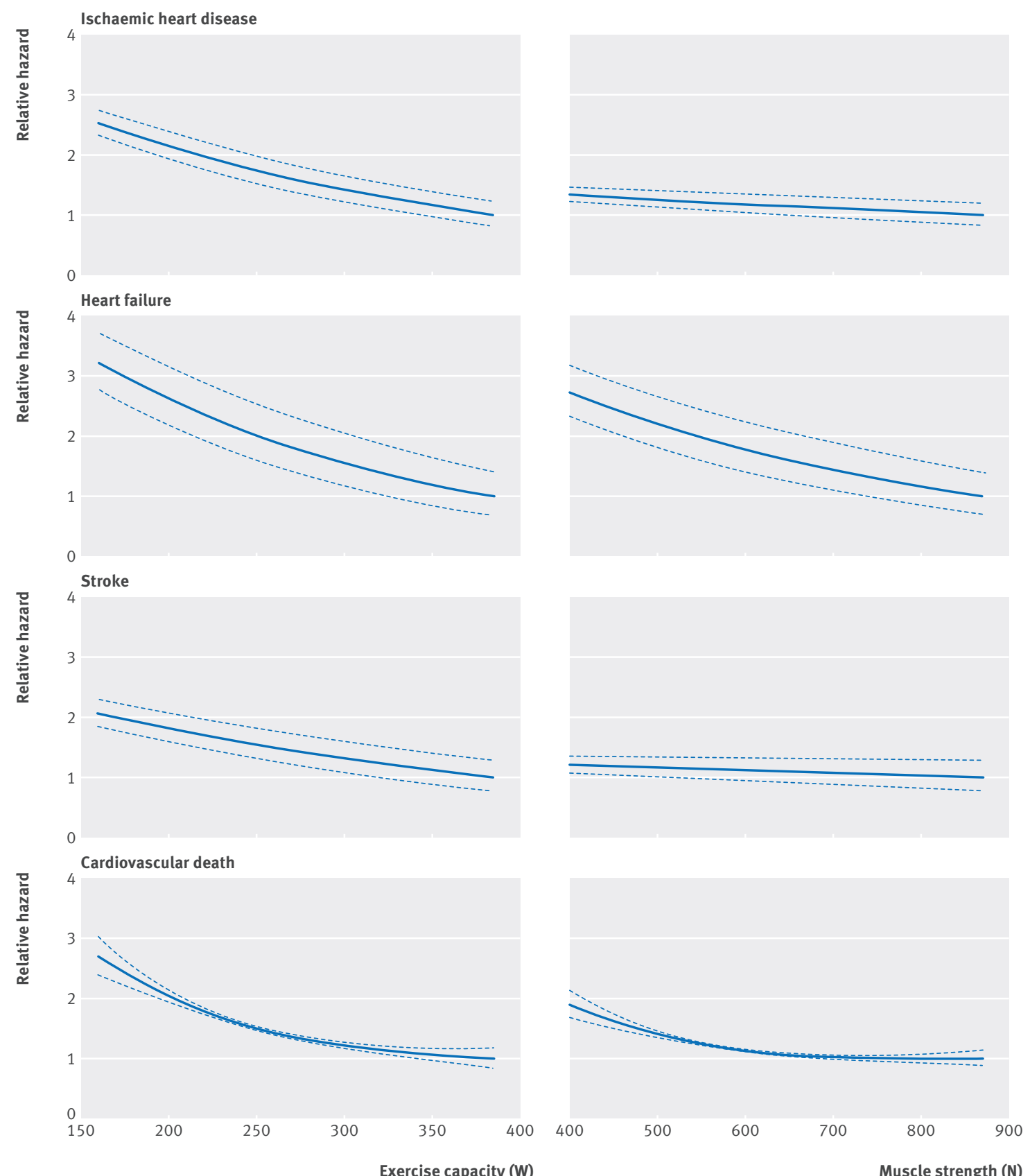

Fig 3 Relations of exercise capacity and muscle strength to risk of subgroups of vascular disease. Solid line=relative hazards; dashed lines $=95 \%$ confidence interval limits. Lines are based on a multivariable regression spline of Cox proportional hazards. Model B (direct effect) is shown, adjusted for age, conscription date, region, education level, height, muscle strength or exercise capacity (muscle strength adjusted for exercise capacity, and vice versa), systolic and diastolic blood pressure, and weight. Only observations between 1 and 99 percentiles are shown

and increased use of autopsies. The young participant age at inclusion minimised the risk of reverse causation caused by pre-existing cardiac disease, although it limited the follow-up to early events.

The study had some limitations. The cohort included only 18 year old men who were mainly white, and generalisability to women, other races, or age groups is unknown. Furthermore, exercise capacity and muscle strength were only measured at the time of conscription, and the applicability of those measures to exposures before and after the conscription is uncertain; any changes in those measures would tend to bias findings towards the null. Other factors linked to the exposure (for example, genetic factors or exercise factors in childhood) rather than the amount of exercise in later life could have contributed to the associations. Studies have suggested that physically active children also are more active as adults, ${ }^{20}$ and changes in levels of physical activity later in life affect risk of cardiovascular events. ${ }^{2122}$

Another limitation was that both exercise capacity and muscle strength were related to body height and 


\begin{tabular}{|c|c|c|c|c|}
\hline & $\begin{array}{l}\text { Low exercise } \\
\text { capacity and low } \\
\text { muscle strength }\end{array}$ & $\begin{array}{l}\text { Low exercise } \\
\text { capacity and high } \\
\text { muscle strength }\end{array}$ & $\begin{array}{l}\text { High exercise } \\
\text { capacity and low } \\
\text { muscle strength }\end{array}$ & $\begin{array}{l}\text { High exercise } \\
\text { capacity and high } \\
\text { muscle strength }\end{array}$ \\
\hline No at risk & 324416 & 223530 & 216716 & 328643 \\
\hline \multicolumn{5}{|l|}{ Vascular disease } \\
\hline No of events & 9512 & 6798 & 3630 & 5715 \\
\hline Incidence* & 10.9 (10.7 to 11.2) & 11.0 (10.7 to 11.2$)$ & 6.6 (6.4 to 6.8$)$ & 6.8 (6.6 to 7.0$)$ \\
\hline Model At & 1.00 (ref) & 0.96 (0.93 to 0.99) & 0.84 (0.81 to 0.87$)$ & 0.85 (0.82 to 0.88$)$ \\
\hline Model B $\ddagger$ & 1.00 (ref) & 0.84 (0.81 to 0.87$)$ & 0.75 (0.73 to 0.79$)$ & 0.67 (0.65 to 0.70$)$ \\
\hline \multicolumn{5}{|l|}{ Arrhythmia } \\
\hline No of events & 4867 & 4329 & 2810 & 5092 \\
\hline Incidence* & $5.6(5.4$ to 5.8$)$ & $6.4(6.2$ to 6.6$)$ & 5.1 (4.9 to 5.3) & 6.1 (5.9 to 6.3) \\
\hline Model At & 1.00 (ref) & 0.99 (0.95 to 1.03) & 0.99 (0.95 to 1.04) & 1.05 (1.01 to 1.10$)$ \\
\hline Model B $\ddagger$ & 1.00 (ref) & 0.91 (0.87 to 0.95) & $0.95(0.90$ to 1.00$)$ & $0.92(0.88$ to 0.97$)$ \\
\hline
\end{tabular}

Data are hazard ratio ( $95 \%$ confidence interval), unless stated otherwise. Ref=reference.

*Per 10000 person years at risk.

†Adjusted for age, conscription date, region, education level, and height.

¥Additionally adjusted for systolic and diastolic blood pressure, weight, and ischaemic heart disease (for arrhythmia outcomes only). and graded associations that we observed with exercise capacity and several subgroups of vascular disease support a causal association. Several mechanisms have been proposed, including better insulin sensitivity, lipid profile, body composition, blood pressure, and autonomic balance. ${ }^{6}$

In children and adolescents, cardiorespiratory fitness has also been related to a reduced incidence of obesity, improved insulin resistance, and lower incidence of cardiovascular risk factors. ${ }^{24-26}$ Several studies have also shown associations of higher muscle strength with lower risk of all cause mortality and vascular disease. ${ }^{16}$ It has been speculated that the effect is mediated through a reduced incidence of abdominal adiposity, weight gain, insulin resistance, metabolic syndrome, hypertension, and chronic inflammation. ${ }^{16} 27$ Previous studies in this group of adolescents ${ }^{2829}$ did not account for the interplay between exercise capacity and muscle strength or assess non-linear associations.

It is unknown whether different modes of trainingfor example, endurance and strength training-differ in their potential for causing arrhythmia. The associations of exercise capacity and muscle strength with risk of arrhythmia are important. Endurance trained athletes with underlying cardiac disease have a higher risk of potentially fatal arrhythmia during sports activity. ${ }^{7}$ Furthermore, athletes are at higher risk of atrial fibrillation and bradyarrhythmia, ${ }^{8-12}$ which might be related to increased pulmonary vein ectopy, vagal tone, pressure or volume load and atrial stretch, dilatation, and fibrosis, alone or in combination. ${ }^{8}$

Most studies have been small, case-control studies comparing active athletes with sedentary people, and lack objective measures of cardiorespiratory fitness. The present study extends those observations to the entire spectrum of cardiorespiratory fitness, using a direct measure of exercise capacity. Of note, to capture only clinically relevant bradyarrhythmia (potentially requiring a pacemaker), sinus bradycardia and grade I atrioventricular block were not part of this outcome. Importantly, although we found an association of exercise capacity with incidence of atrial fibrillation or flutter in the present study, it did not translate into an increased risk of stroke. Furthermore, we did not find a higher risk of ventricular arrhythmia with higher exercise capacity.

The similarity of the independent associations of exercise capacity and muscle strength with vascular disease is noteworthy. It could indicate that different modes of training trigger the same or similar biological responses, or that the two tests capture different aspects of similarly trained people because of factors unrelated to their training. The first interpretation could be disputed by the observation of different cardiac adaptations in athletes of different sports. ${ }^{30}$ These mechanisms may be related to risk of arrhythmia, but mechanisms other than cardiac geometry could be more important for atherosclerotic vascular disease, as outlined above.

The present cohort has only been followed to median age of 44.6 years, and it is worth asking whether the trend continues up to older ages. For vascular disease, 


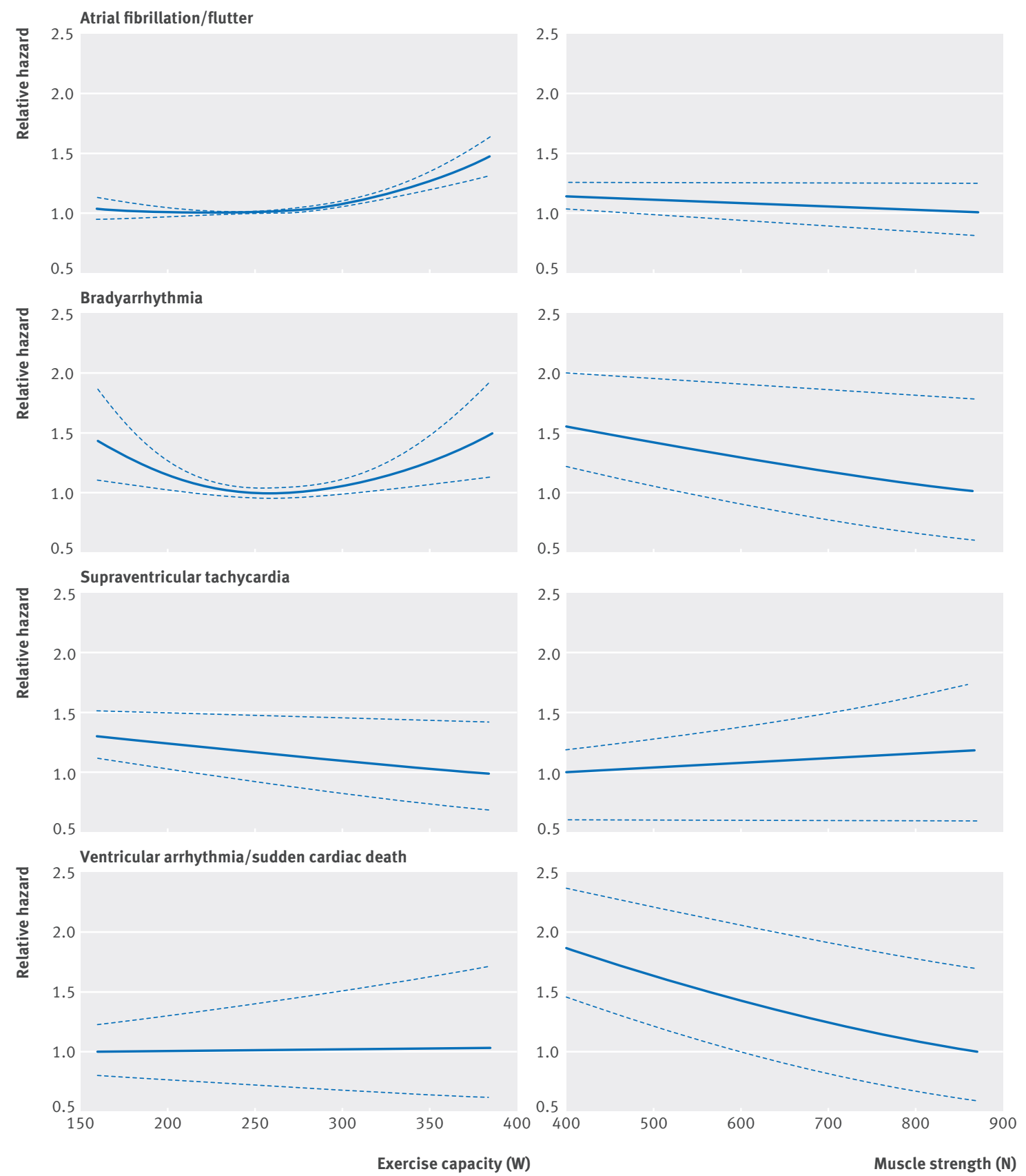

Fig 4 | Relations of exercise capacity and muscle strength to risk of subgroups of arrhythmia. Solid line=relative hazards; dashed lines $=95 \%$ confidence interval limits. Lines are based on a multivariable regression spline of Cox proportional hazards. Model B (direct effect) are shown, adjusted for age, conscription date, region, education level, height, muscle strength or exercise capacity (muscle strength adjusted for exercise capacity, and vice versa), systolic and diastolic blood pressure, weight, and ischaemic heart disease. Only observations between 1 and 99 percentiles are shown

several studies have suggested good fitness at a young age to have a long term protective effect, even if participants change fitness status between measurements. 223132 In the present study, this trend appears continuous at least until age 57 years (fig 1). For arrhythmia, particularly atrial fibrillation, it is possible that the trend of higher incidence with higher exercise capacity will diminish with higher age. In older people, atrial fibrillation is closely associated with vascular disease risk factors such as hypertension and obesity. Consequently, some studies of older people have suggested a reduced risk of atrial fibrillation with increased physical activity levels. ${ }^{12}{ }^{33}$ Any such transition did not seem to appear before age 57 years in the present cohort (fig 1).

\section{Conclusions}

In the present study, higher exercise capacity and higher muscle strength in late adolescence were independently associated with lower risk of subsequent vascular disease in this large cohort of young men. We observed a U shaped association of exercise capacity with arrhythmia, driven by a direct association with risk 
of atrial fibrillation or flutter and by a U shaped association with bradyarrhythmia. Higher muscle strength was associated with lower risk of arrhythmia, driven by a lower risk of bradyarrhythmia and ventricular arrhythmia. The combined associations of high exercise capacity and high muscle strength versus low exercise capacity and low muscle strength with lower risk of vascular disease were prominent. The lower risk of vascular events with higher exercise capacity did not seem to be outweighed by increased risk of arrhythmia. Generalisability to women is unknown and equivalent data for women are needed.

Contributors: KA and JS conceived and coordinated the investigations. KA wrote the first draft of the manuscript. FR and PT were responsible for the preparation of data. All the authors undertook revisions and contributed intellectually to the development of this paper. KA is the study guarantor.

Funding: IS was funded by the Swedish Research Council (grants 2007-5942 and 2010-1078). KA received a grant from the Geriatric Fund, Sweden. MN was funded by the Swedish Research Council (grant K2014-99X-22495-01-3). All researchers were independent from funders. Competing interests: All authors have completed the ICMJE uniform disclosure form at www.icmje.org/coi_disclosure.pdf and declare: no support from any organisation for the submitted work; $J \mathrm{~S}$ is part of the advisory board for Itrim and AstraZeneca; $\mathrm{MN}$ is part of the advisory board for Itrim; no financial relationships with any organisations that might have an interest in the submitted work in the previous three years. Ethical approval: The study protocol was approved by the regional ethical review board at Karolinska Institutet, Stockholm, Sweden.

Data sharing: Additional data regarding technical details, statistical code, and derived data are available from the principal investigator at kasper.andersen@medsci.uu.se. Consent was not obtained but the presented data are anonymised and risk of identification is low.

The lead author KA (the manuscript's guarantor) affirms that the manuscript is an honest, accurate, and transparent account of the study being reported; that no important aspects of the study have been omitted; and that any discrepancies from the study as planned (and, if relevant, registered) have been explained.

This is an Open Access article distributed in accordance with the Creative Commons Attribution Non Commercial (CC BY-NC 4.0) license, which permits others to distribute, remix, adapt, build upon this work non-commercially, and license their derivative works on different terms, provided the original work is properly cited and the use is noncommercial. See: http://creativecommons.org/licenses/by-nc/4.0/.

1 Lozano R, Naghavi M, Foreman K, et al. Global and regional mortality from 235 causes of death for 20 age groups in 1990 and 2010: a systematic analysis for the Global Burden of Disease Study 2010. Lancet 2012;380:2095-128.

2 Friberg L, Bergfeldt L. Atrial fibrillation prevalence revisited. J Intern Med 2013:274:461-8.

3 Nocon M, Hiemann T, Muller-Riemenschneider F, et al. Association of physical activity with all-cause and cardiovascular mortality: a systematic review and meta-analysis. Eur J Cardiovasc Prev Rehabil 2008;15:239-46.

4 Sofi F, Capalbo A, Cesari F, et al. Physical activity during leisure time and primary prevention of coronary heart disease: an updated meta-analysis of cohort studies. Eur / Cardiovasc Prev Rehabil 2008:15:247-57.

5 Held C, Iqbal R, Lear SA, et al. Physical activity levels, ownership of goods promoting sedentary behaviour and risk of myocardial infarction: results of the INTERHEART study. Eur Heart J 2012;33:452-66.

6 Lee DC, Artero EG, Sui X, et al. Mortality trends in the general population: the importance of cardiorespiratory fitness. Psychopharmacol 2010;24(4 suppl):27-35.

7 Corrado D, Schmied C, Basso C, et al. Risk of sports: do we need a pre-participation screening for competitive and leisure athletes? Eur Heart J 2011:32:934-44.

8 Mont L, Elosua R, Brugada J. Endurance sport practice as a risk factor for atrial fibrillation and atrial flutter. Europace 2009:11:11-7.

9 Andersen K, Farahmand B, Ahlbom A, et al. Risk of arrhythmias in 52 755 long-distance cross-country skiers: a cohort study. Eur Heart J 2013;34:3624-31.
10 Claessen G, Colyn E, La Gerche A, et al. Long-term endurance sport is a risk factor for development of lone atrial flutter. Heart 2011:97:918-22.

11 Baldesberger S, Bauersfeld U, Candinas R, et al. Sinus node disease and arrhythmias in the long-term follow-up of former professional cyclists. Eur Heart/ 2008:29:71-8.

12 Drca N, Wolk A, Jensen-Urstad M, et al. Atrial fibrillation is associated with different levels of physical activity levels at different ages in men Heart 2014;100:1037-42.

13 Heimburger O, Qureshi AR, Blaner WS, et al. Hand-grip muscle strength, lean body mass, and plasma proteins as markers of nutritional status in patients with chronic renal failure close to start of dialysis therapy. Am J Kid Dis 2000;36:1213-25.

14 Shin H, Liu PY, Panton LB, et al. Physical performance in relation to body composition and bone mineral density in healthy, overweight, and obese postmenopausal women. J Geriatr Phys Ther 2014:37:7-16

15 Taaffe DR, Cauley JA, Danielson M, et al. Race and sex effects on the association between muscle strength, soft tissue, and bone mineral density in healthy elders: the Health, Aging, and Body Composition Study. J Bone Miner Res 2001;16:1343-52.

16 Artero EG, Lee DC, Lavie C], et al. Effects of muscular strength on cardiovascular risk factors and prognosis. / Cardiopulm Rehabil Prev 2012:32:351-8.

17 Royston P, Sauerbrei W. A new approach to modelling interactions between treatment and continuous covariates in clinical trials by using fractional polynomials. Stat Med 2004:23:2509-25.

18 Ludvigsson JF, Andersson E, Ekbom A, et al. External review and validation of the Swedish national inpatient register. BMC Public Health 2011:11:450.

19 Baturova MA, Lindgren A, Carlson J, et al. Atrial fibrillation in patients with ischaemic stroke in the Swedish national patient registers: how much do we miss? Europace 2014;16:1714-9.

20 Telama R. Tracking of physical activity from childhood to adulthood: a review. Obes Facts 2009;2:187-95.

21 Erikssen G, Liestol K, Bjornholt J, et al. Changes in physical fitness and changes in mortality. Lancet 1998:352:759-62.

22 Blair SN, Kohl HW, 3rd, Barlow CE, et al. Changes in physical fitness and all-cause mortality. A prospective study of healthy and unhealthy men. JAMA 1995;273:1093-8.

23 Neovius M, Sundström J, Rasmussen F. Combined effects of overweight and smoking in late adolescence on subsequent mortality: nationwide cohort study. BMJ 2009;338:b496.

24 Ortega FB, Ruiz IR, Castillo MJ, et al. Physical fitness in childhood and adolescence: a powerful marker of health. Int J Obes 2008;32:1-11.

25 Lee S, Bacha F, Gungor N, et al. Cardiorespiratory fitness in youth: relationship to insulin sensitivity and beta-cell function. Obesity 2006;14:1579-85.

26 Berman LJ, Weigensberg MJ, Spruijt-Metz D. Physical activity is related to insulin sensitivity in children and adolescents, independent of adiposity: a review of the literature. Diabetes Metab Res Rev 2012;28:395-408

27 Andersen K, Pedersen BK. The role of inflammation in vascular insulin resistance with focus on IL-6. Horm Metab Res 2008;40:635-9.

28 Silventoinen K, Magnusson PK, Tynelius P, et al. Association of body size and muscle strength with incidence of coronary heart disease and cerebrovascular diseases: a population-based cohort study of one million Swedish men. Int I Epidemiol 2009:38:110-8.

29 Ortega FB, Silventoinen K, Tynelius P, et al. Muscular strength in male adolescents and premature death: cohort study of one million participants. BMI 2012:345:e7279

30 Pluim BM, Zwinderman AH, van der Laarse A, et al. The athlete's heart. A meta-analysis of cardiac structure and function. Circulation 2000;101:336-44

31 Lee DC, Pate RR, Lavie Cl, et al. Leisure-time running reduces all-cause and cardiovascular mortality risk. I Am Coll Cardiol 2014;64:472-81

32 Lee DC, Sui X, Artero EG, et al. Long-term effects of changes in cardiorespiratory fitness and body mass index on all-cause and cardiovascular disease mortality in men: the Aerobics Center Longitudinal Study. Circulation 2011;124:2483-90.

33 Mozaffarian D, Furberg CD, Psaty BM, et al. Physical activity and incidence of atrial fibrillation in older adults: the cardiovascular health study. Circulation 2008;118:800-7.

(c) BMJ Publishing Group Ltd 2015

Web appendix 1: Complete list of ICD diagnoses used in the study

Web appendix 2: Web figures

Web appendix 3: Web tables 